\title{
Incidence of long term backache was similar between women receiving epidural analgesia during labour and those who did not
}

\author{
Howell CJ, Kidd C, Roberts Wet al. A randomised controlled trial of epidural compared with non-epidural analgesia in \\ labour. BJOG 2001 Jan;108:27-33.
}

\section{QUESTION: In women during labour, does epidural analgesia lead to long term backache more than non-epidural analgesia?}

Design

Randomised (allocation concealed*), unblinded,* controlled trial with 12 months follow up.

\section{Setting}

Maternity unit of a district general hospital in Staffordshire, UK.

\section{Patients}

369 women (mean age 24 y) at first pregnancy who were in spontaneous, full term labour, had a normal obstetric and medical history, had no contraindication to either form of analgesia, were willing to participate, and had continued eligibility at time of labour. Follow up was $85 \%$ at 3 months and $88 \%$ at 12 months.

\section{Intervention}

Women were allocated to epidural analgesia $(n=184)$ or non-epidural analgesia $(\mathrm{n}=185)$. Epidural analgesia consisted of $0.25 \%$ bupivacaine $(10 \mathrm{ml})$ with top ups of 5 to $10 \mathrm{ml}$ of $0.25 \%$ bupivacaine by the midwife as required. Non-epidural analgesia consisted of 50 to 100 $\mathrm{mg}$ of intramuscular pethidine, which could be repeated according to standard practice. Entonox was available to both groups. Management of labour was according to standard protocols.

Sources of funding: WellBeing and North Staffordshire Medical Institute.

For correspondence: Mr R B Johanson, Academic Department of Obstetrics and Gynaecology, North Staffordshire Hospital (NHS) Trust, Newcastle Road, Stoke on Trent, Staffordshire

ST4 6OG, UK. Fax +44 (0)1782552695.

\section{Main outcome measures}

Incidence of long term backache. Secondary outcomes were operative delivery rates and maternal satisfaction. All outcomes were measured using a maternal health questionnaire.

\section{Main results}

Analysis was by intention to treat. 61 women $(33 \%)$ in the epidural group did not receive the allocated epidural, and 52 women (28\%) in the non-epidural group received an epidural. No differences existed in middle or lower back pain at either 3 or 12 months after

Epidural v non-epidural analgesia during labour for backache at 3 and 12 months†

\begin{tabular}{lrlll} 
Outcomes & Follow up & Event rates & RRI (95\% Cl) & NNH \\
Low backache & 3 months & $35 \% v 34 \%$ & $0.4 \%(-26$ to 36$)$ & Not significant \\
\hline & 12 months & $35 \% v 27 \%$ & $28 \%(-7.3$ to 79$)$ & Not significant \\
\hline Middle backache & 3 months & $22 \% v 20 \%$ & $8.7 \%(-29$ to 68$)$ & Not significant \\
\hline & & & RRR (CI) & NNT \\
& 12 months & $16 \% v 16 \%$ & $1.0 \%(-63$ to 40$)$ & Not significant \\
\hline
\end{tabular}

†Abbreviations defined in glossary; RRR, RRI, NNT, NNH, and $\mathrm{Cl}$ calculated from data in article.

delivery (table). In the epidural group, duration of second stage labour was increased by 19 minutes $(95 \%$ CI 6.5 to $30.9, \mathrm{p}=0.003)$ and the rate of instrumental delivery was higher (30\% [epidural] v 19\% [non-epidural], $\mathrm{p}=0.03$ ). Caesarean section rates were similar between groups. Maternal satisfaction with the experience of childbirth and pain relief was similarly high in both groups.

\section{Conclusion}

Incidence of long term backache was similar among women receiving epidural analgesia and those not receiving epidural analgesia during labour.

*See glossary.

\section{COMMENTARY}

Epidural analgesia during labour is an effective method of relieving pain and has been used extensively since the mid1970 s, even in developing countries. Unfortunately, it has been widely incorporated into practice without enough evidence about its possible benefits and side effects. It is therefore not surprising that epidural analgesia has been blamed for various adverse events. This study by Howell $e t a l$ is the first randomised controlled trial (RCT) to specifically address the issue of post-delivery backache.

A limitation of this trial is that we may not be able to generalise the results because only $31 \%$ ( 875 of 2840 ) of all pregnant women attending the antenatal clinic were willing to participate. Of these, another 506 women withdrew in labour before randomisation. Thus, only $42 \%$ (369 of 875) of women were eventually randomly allocated. A further problem was that nearly a third of the women in each group did not receive the allocated treatment, which reduces the power of the study.

Women in labour who request pain relief should be fully informed about the benefits and possible risks of their choice of analgesia. ${ }^{1}$ There are still many unanswered questions about different pain relief methods (eg, effect on fetus or neonate), which need to be addressed with further RCTs.

This study provides evidence to show that epidural analgesia is not a significant factor in causing postpartum backache. Women who have epidural analgesia are still satisfied with their overall experience of labour.

Cheryl Nikodem, DCur ECRU/University of the Witwatersrand Johannesburg, South Africa

1 Howell CJ. Epidural versus non-epidural analgesia for pain relief in labour. Cochrane Database Syst Rev 2001;(2):CD000331. 